\title{
Carotid Artery Tortuosity Is Associated with Connective Tissue Diseases
}

\author{
(D).P. Welby, (DS.T. Kim, (D) C.M. Carr, (D) V.T. Lehman, (D).H. Rydberg, (D).T. Wald, (DP.H. Luetmer, (DD.M. Nasr, and \\ (D) W. Brinjikji
}

\begin{abstract}
BACKGROUND AND PURPOSE: There is a general assumption in the cerebrovascular literature that there is an association between carotid artery tortuosity and connective tissues disease; however, this has not been firmly established. The purpose of this study was to determine the prevalence of carotid artery tortuosity in patients with connective tissue diseases relative to matched controls.
\end{abstract}

MATERIALS AND METHODS: Patients with previous CTA or MRA and a diagnosis of connective tissue diseases were identified and compared with a cohort of age-matched controls. Radiologists blinded to the diagnosis reviewed the images and evaluated the presence of carotid artery tortuosity (including loops, kinks, or coils). Continuous variables were compared using the Student $t$ test, and categoric variables with $\chi^{2}$ tests.

RESULTS: One hundred forty-three patients with connective tissue disease and 143 controls were included in this study. Specific diagnoses included Marfan ( $n=33)$, nonvascular Ehlers-Danlos $(n=36)$, Ehlers-Danlos vascular-type $(n=32)$, neurofibromatosis type $1(n=26)$, and Loeys-Dietz $(n=16)$ syndromes. The presence of carotid tortuosity was $44 \%$ in connective tissue disease and $16 \%$ in controls $(P<.001)$. Of tortuosity manifestations, coils were most prevalent $(23 \%$ versus $3 \% ; P<.001)$. Among the various connective tissue diseases, the rates of any carotid tortuosity were $88 \%$ for Marfan syndrome, $63 \%$ for Loeys-Dietz syndrome, $42 \%$ for neurofibromatosis type 1 , and $19 \%$ for both vascular- and nonvascular-type Ehlers-Danlos syndrome. The positive predictive value of the combination of aortic aneurysm and carotid tortuosity being associated with connective tissue disease was $95.4 \%$. The specificity was $98.6 \%$.

CONCLUSIONS: Carotid artery tortuosity is highly associated with connective tissue diseases, particularly Marfan syndrome, Loeys-Dietz syndrome, and neurofibromatosis type 1. Such findings are relevant in risk assessment for vascular complications in connective tissue disease, endovascular treatment planning, and in understanding the pathomechanisms of vascular tortuosity in general.

ABBREVIATIONS: $\mathrm{CTD}=$ connective tissue disease; $\mathrm{EDS}=$ Ehlers-Danlos syndrome; LDS = Loeys-Dietz syndrome; NF1 = neurofibromatosis type 1

C arotid artery tortuosity is defined as vascular elongation leading to redundancy or an altered course. Recent evidence suggests that the prevalence of carotid tortuosity is higher than conventionally expected ranging from $18 \%$ to $34 \%$. ${ }^{1,2}$ While often an incidental finding, carotid tortuosity has been known to contribute to cases of vertigo, tinnitus, and stroke secondary to

Received May 5, 2019; accepted after revision July 31.

From the Mayo Clinic Alix School of Medicine (J.P.W.) and Departments of Radiology (C.M.C., V.T.L., C.H.R., J.T.W., P.H.L., W.B.), Neurology (D.M.N.), and Neurosurgery (W.B.), Mayo Clinic, Rochester, Minnesota; and Department of Radiology (S.T.K.), University of California, San Francisco, San Francisco, California. Please address correspondence to John P. Welby, MD, Mayo Clinic Alix School of Medicine, 200 lst St SW, Rochester, MN 55905; e-mail: Welby.John@mayo.edu

Indicates article with supplemental on-line tables.

http://dx.doi.org/10.3174/ajnr.A6218 dissection. ${ }^{2,3}$ Furthermore, carotid tortuosity has been known to complicate access to the cranial vasculature in neuroendovascular procedures and has anecdotally been associated with a higher risk of iatrogenic dissection. ${ }^{4}$

The mechanisms underlying carotid artery tortuosity remain unclear. ${ }^{5}$ Studies suggest that tortuous carotid arteries exhibit histopathologic changes, including degeneration of the tunica media and elastic lamina. ${ }^{6}$ Reported risk factors include hypertension, elevated body mass index, advanced age, and atherosclerotic disease. ${ }^{3,7-9}$ It is often assumed that patients with tortuosity may have some underlying collagenopathy as well; however, to our knowledge this has yet to be studied in connective tissue diseases (CTDs). Previous study has demonstrated a relationship between CTDs and neurovascular abnormalities. Through varied mechanisms such as altered fibrillin 1 structure in Marfan syndrome, 


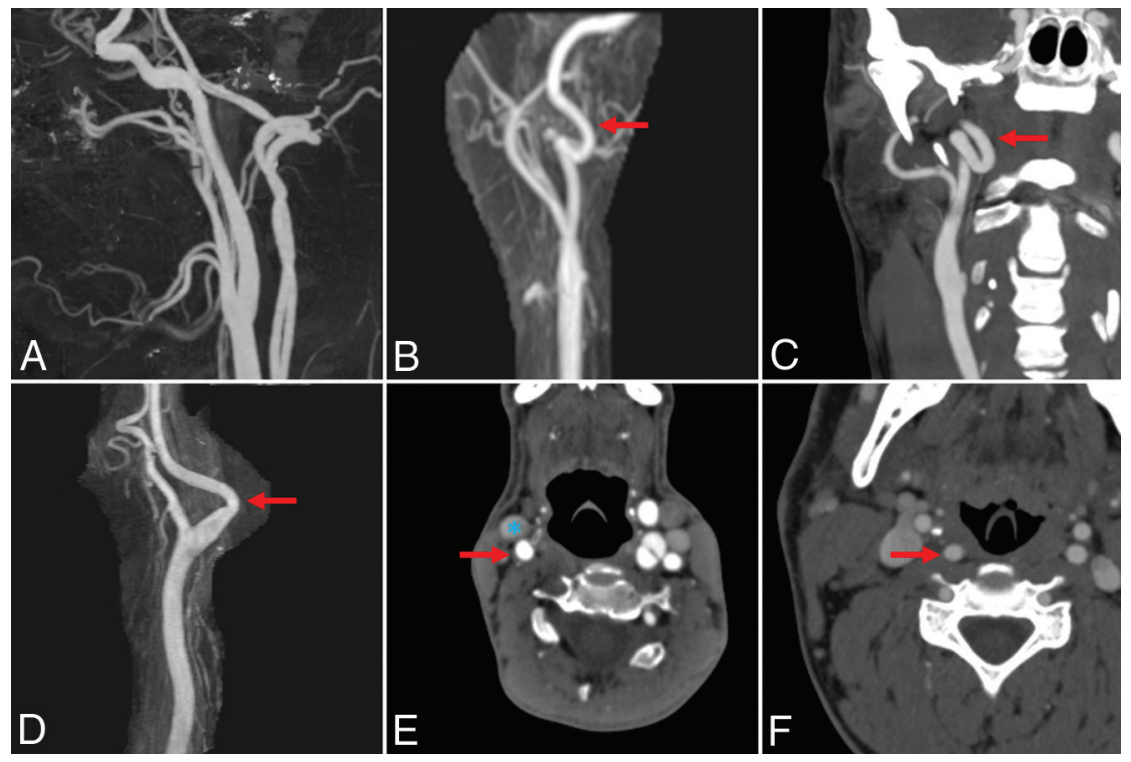

FIGURE. Examples of carotid artery tortuosity and path alterations. A, Sagittal CTA reconstruction shows normal bilateral carotid and vertebral arteries. $B$, Anterior reconstruction MRA of a patient with Marfan syndrome shows a carotid loop (red arrow). C, Coronal MIP CTA of a patient with Marfan syndrome shows bilateral carotid coils (red arrow). D, Sagittal-reconstruction MRA of a patient with EDS shows a carotid artery (red arrow) kink. E, Axial CTA of a patient with LDS shows a retrojugular carotid artery (carotid artery, red arrow; jugular vein, blue asterisk). F, Axial CTA of a patient with Marfan syndrome demonstrates a retropharyngeal carotid artery (red arrow) course.

collagen mutations in Ehlers-Danlos syndrome (EDS), or transforming growth factor- $\beta$ signaling abnormalities in Loeys-Dietz syndrome (LDS), CTDs are demonstrated to alter vessel makeup and lead to vascular abnormalities, including aneurysms and dissections. ${ }^{10}$ To date, no studies have rigorously determined whether CTDs are, in fact, associated with carotid artery tortuosity. Demonstrating this association could be important for identifying patients who could benefit from screening for CTDs as well as understanding the pathomechanisms behind cervical carotid tortuosity. To better understand whether there is an association between CTDs and carotid artery tortuosity, we performed an age- and sex-matched case-control study of patients with and without CTD including Marfan syndrome, EDS, neurofibromatosis type 1 (NF1), and LDS, and carotid artery tortuosity.

\section{MATERIALS AND METHODS \\ Patient Identification}

Following institutional review board approval, we identified all patients with prior CTA or MRA of the cervical vasculature and a history of CTD, including Marfan syndrome, EDS, LDS, or NF1 from July 1998 to January 2017. All patients had a clinically confirmed diagnosis of these syndromes as determined by a medical geneticist at Mayo Clinic. Patients who had other forms of unconfirmed CTDs were excluded; any patients with a history or suspicion of other connective tissue diseases (ie, spontaneous coronary artery dissection and so forth) were excluded from the control group. We then identified a group of 143 age-matched controls who had carotid
CTA or MRA performed as part of emergency department work-ups for various indications, including trauma, stroke, dizziness, vertigo, and so forth.

\section{Imaging Analysis}

Radiologists blinded to each patient's medical history reviewed the CTA or MRA imaging to evaluate the presence of tortuosity within the cervical segment of the internal carotid artery per previously described methods. ${ }^{2}$ Radiologists had access to multiplanar reformatted images and 3D reconstructions as available. Carotid artery tortuosity was defined as the presence of shape deformations including coils, loops, and kinks. Specifically, shape deformations were assessed using the following criteria: 1) The presence of loops was defined by findings of C- or $\mathrm{S}$-shaped deformities wherein the vessel exhibited 2 turns with angles of $\leq 90^{\circ}$; 2) coils exhibited complete, $360^{\circ}$, turns in the vessel; and 3) kinks were observed as singular $\geq 90^{\circ}$ bends in the vessel. Furthermore, we explored path alterations including retrojugular and retropharyngeal anatomy. Path alterations were defined by the following criteria: 1) A retrojugular carotid path was defined as $>50 \%$ of the internal carotid artery by cross-sectional area traveling posterior to the internal jugular vein; and 2) a retropharyngeal path was defined as $>50 \%$ of the ICA traveling behind (medially to) the lateral edge of the pharynx. Examples of tortuosity and path alterations can be found in the Figure. Additionally, the presence of atherosclerotic changes and fibromuscular dysplasia was assessed.

\section{Clinical Variables}

Secondary clinical and demographic information including age and sex were pulled from patient charts as available. Diagnoses of hypertension, dyslipidemia (including hyperlipidemia, hypercholesterolemia, and hypertriglyceridemia), diabetes, smoking history, stroke history, coronary artery disease history, and other history of dissection, aortic root dilation, or aneurysm were identified using the medical record search function and assessed categorically by the presence or nonpresence in physician notes and radiologic reads. Common synonyms and abbreviations were included.

\section{Sensitivity Analysis}

In addition to studying the association between CTD and carotid tortuosity, we also examined the association between the combination of both an aortic dilation/aneurysm and carotid tortuosity and connective tissue diseases.

AJNR Am J Neuroradiol 40:1738-43 Oct 2019 www.ajnr.org 
Table 1: Demographics

\begin{tabular}{lcccccc}
\hline & \multicolumn{2}{c}{ CTD } & & \multicolumn{3}{c}{ Control } \\
\cline { 2 - 3 } \cline { 5 - 6 } & Mean & SD & & Mean & SD & $\boldsymbol{P}$ \\
\hline Age (yr) & 42.19 & 16.48 & & 38.84 & 20.14 & 0.13 \\
\cline { 2 - 6 } & No. & $\%$ & No. & $\%$ & \\
\cline { 2 - 3 } Sex (female) & 92 & $64.3 \%$ & 102 & $71.3 \%$ & 0.21 \\
Imaging technique & & & & & \\
CTA & 42 & $39.4 \%$ & & 143 & $100 \%$ & \\
MRA & 116 & $81.1 \%$ & 44 & $30.8 \%$ & \\
\hline
\end{tabular}

Table 2: Baseline characteristics

\begin{tabular}{|c|c|c|c|c|c|}
\hline & \multicolumn{2}{|c|}{$\begin{array}{c}\text { CTD } \\
(n=143)\end{array}$} & \multicolumn{2}{|c|}{$\begin{array}{l}\text { Control } \\
(n=143) \\
\end{array}$} & \multirow[b]{2}{*}{$P$} \\
\hline & No. & $\%$ & No. & $\%$ & \\
\hline Dyslipidemia & 30 & $21 \%$ & 31 & $22 \%$ & .89 \\
\hline HTN & 38 & $27 \%$ & 43 & $30 \%$ & .51 \\
\hline DM & 3 & $2 \%$ & 15 & $10 \%$ & $.004^{\mathrm{a}}$ \\
\hline Smoking & 44 & $31 \%$ & 50 & $35 \%$ & .45 \\
\hline Stroke history & 20 & $14 \%$ & 21 & $15 \%$ & .87 \\
\hline CAD history & 8 & $6 \%$ & 15 & $10 \%$ & .13 \\
\hline $\begin{array}{l}\text { Other aneurysm/dis- } \\
\text { section/ } \\
\text { FMD history }\end{array}$ & 85 & $59 \%$ & 28 & $20 \%$ & $<.001^{\mathrm{a}}$ \\
\hline CNS & 12 & $8 \%$ & 8 & $6 \%$ & .35 \\
\hline Carotid & 19 & $13 \%$ & 3 & $2 \%$ & $<.001^{\mathrm{a}}$ \\
\hline Vertebral & 11 & $8 \%$ & 5 & $3 \%$ & .12 \\
\hline Aortic & 58 & $41 \%$ & 7 & $5 \%$ & $<.001^{\mathrm{a}}$ \\
\hline Subclavian/brachial & 5 & $3 \%$ & 0 & $0 \%$ & $.02^{\mathrm{a}}$ \\
\hline Coronary & 8 & $6 \%$ & 0 & $0 \%$ & $.004^{\mathrm{a}}$ \\
\hline $\begin{array}{l}\text { Abdominal } \\
\text { (mesenteric, celiac, } \\
\text { splenic, hepatic) }\end{array}$ & 18 & $13 \%$ & 0 & $0 \%$ & $<.001^{\mathrm{a}}$ \\
\hline Renal & 10 & $7 \%$ & 0 & $0 \%$ & $.001^{\mathrm{a}}$ \\
\hline Iliac/femoral/popliteal & 15 & $10 \%$ & 0 & $0 \%$ & $<.001^{a}$ \\
\hline
\end{tabular}

Note:-DM indicates diabetes mellitus; FMD, fibromuscular dysplasia; HTN, hypertension; CAD, coronary artery disease.

a Significant.

\section{Statistical Analysis}

Obtained data were analyzed using JMP, Version 13 (SAS Institute, Cary, North Carolina, 1989-2007). Data were analyzed on a per-patient basis. Continuous variables were compared using a 2-tailed Student $t$ test, and categoric variables, using $\chi^{2}$ tests. Multivariate logistic regression analysis was performed on demographic information, clinical variables, and association with tortuosity. Continuous variables are reported as per-unit change. Any ICA tortuosity was defined as the presence of any left or right kink, coil, or loop. Laterality, including the presence of any tortuosity on the left, right, or bilateral carotid arteries, was also included in the analysis. Distinct pathway alterations, including retropharyngeal and retrojugular, were analyzed independently. Statistical significance was determined with a threshold $\alpha$ $P<.05$. We performed subgroup analyses based on each individual connective tissue disease population relative to the control population as well.

\section{RESULTS}

\section{Patient Population and Baseline Characteristics}

We identified 143 patients with CTD (median age, 42 years; interquartile range, $29-54$ years; $64.3 \%$ female) including Marfan syndrome $(n=33)$, EDS $(n=68)$, NF1 $(n=26)$, and LDS $(n=16)$ and available CTA or MRA. These individuals were paired with 143 matched controls without a diagnosis of CTD (median age, 40 years; interquartile range, 22-59 years; $71.3 \%$ female). Univariate analysis did not reveal a statistically significant difference in age or sex of the CTD group relative to controls. A summary of demographic characteristics is provided in Table 1 . In patients with CTD, study indications most commonly included CTD screening (38\%), whereas controls included trauma (20\%) and headache (19\%). A summary of study indications is included in On-line Table 1.

Baseline characteristics including relevant underlying vascular risk factors were assessed (Table 2). Patients with CTD exhibited a nonsignificant difference in the history of smoking ( $31 \%$ versus $35 \% ; P=.45$ ), stroke ( $14 \%$ versus $15 \%$; $P=.87$ ), and coronary artery disease (6\% versus $10 \% ; P=.13$ ), in addition to dyslipidemia ( $21 \%$ versus $22 \%, P=.89)$ and hypertension $(27 \%$ versus $30 \% ; P=.51)$. Controls had an increased prevalence of diabetes ( $2 \%$ versus $10 \% ; P=.004)$. Assessment of other aneurysm or dissection history revealed a significantly increased prevalence of carotid, aortic, subclavian/brachial, renal, and other visceral vascular pathologies in patients with CTD relative to controls.

To explore previously published associations of carotid tortuosity with the above demographic and clinical variables in the general population, we performed analysis on the presence of any ICA tortuosity within the control group alone (On-line Table 2). Age, stroke history, diabetes, dyslipidemia, and hypertension were significantly associated with tortuosity on univariate analysis. On multivariate analysis, smoking history, stroke history, and hypertension were found to be significantly associated with tortuosity.

\section{Carotid Artery Tortuosity}

Comparison of patients with CTD with controls demonstrated significant differences in the prevalence of carotid artery tortuosity between these 2 groups. Patients with CTD exhibited increased prevalence of any ICA tortuosity (44\% versus $16 \%$; OR, $4.11 ; 95 \% \mathrm{CI}, 2.36-7.16 ; P<.001)$, loops $(21 \%$ versus $10 \%$; OR, 2.27; 95\% CI, 1.16-4.42; $P=.015$ ), and coils (23\% versus $3 \%$; OR, 10.43; 95\% CI, 3.59-30.31; $P<.001)$ reaching statistical significance. Patients with CTD had increased prevalence of bilateral tortuosity ( $30 \%$ versus 10\%; OR, 3.67; 95\% CI, 1.93-6.98; $P<.001)$. Of patients with CTD with any ICA tortuosity, bilateral tortuosity was present in 68\% $(n=43 / 63)$. While the presence of any left or right tortuosity was significant in patients with CTD relative to controls, right-sided pathologies were noted to be more common than left-sided ones in patients with CTD ( $40 \%$ versus $34 \%$; $P<.001$ ). No significant difference was found in the prevalence of carotid kinks or alterations in the carotid route, including retrojugular or retropharyngeal paths relative to controls. There was no significant difference in rates of fibromuscular dysplasia ( $3 \%$ versus $1 \% ; P=.25$ ); however, there was a significantly lower rate of atherosclerosis in patients with CTD (5\% versus 26\%; $P<.001$; Table 1).The aforementioned findings are summarized in Table 3.

To better explore the extent of the association of CTD status and carotid artery tortuosity, we performed multivariate 


\begin{tabular}{|c|c|c|c|c|c|c|}
\hline & \multicolumn{2}{|c|}{ CTD $(n=143)$} & \multicolumn{2}{|c|}{ Controls $(n=143)$} & \multirow[b]{2}{*}{ OR (95\% Cl) } & \multirow[b]{2}{*}{$P$} \\
\hline & No. & $\%$ & No. & $\%$ & & \\
\hline Any ICA tortuosity & 63 & $44 \%$ & 23 & $16 \%$ & $4.11(2.36-7.16)$ & $<.001^{\mathrm{a}}$ \\
\hline Bilateral & 43 & $30 \%$ & 15 & $10 \%$ & 3.67 (1.93-6.98) & $<.001^{\mathrm{a}}$ \\
\hline Left & 49 & $34 \%$ & 21 & $15 \%$ & $3.03(1.70-5.40)$ & $<.001^{\mathrm{a}}$ \\
\hline Right & 57 & $40 \%$ & 17 & $12 \%$ & $4.91(2.68-9.01)$ & $<.001^{\mathrm{a}}$ \\
\hline Loop & 30 & $21 \%$ & 15 & $10 \%$ & $2.27(1.16-4.42)^{\prime}$ & $.015^{\mathrm{a}}$ \\
\hline Coil & 33 & $23 \%$ & 4 & $3 \%$ & $10.43(3.59-30.31)$ & $<.001^{\mathrm{a}}$ \\
\hline Kink & 13 & $9 \%$ & 10 & $7 \%$ & $1.33(0.56-3.14)$ & .51 \\
\hline \multicolumn{7}{|l|}{ Pathway alterations } \\
\hline Retrojugular & 36 & $25 \%$ & 29 & $20 \%$ & $1.32(0.76-2.31)$ & .32 \\
\hline Retropharyngeal & 6 & $4 \%$ & 5 & $3 \%$ & $1.21(0.36-4.05)$ & .76 \\
\hline \multicolumn{7}{|l|}{ Other } \\
\hline Any carotid FMD & 5 & $3 \%$ & 2 & $1 \%$ & $2.55(0.49-13.39)$ & .25 \\
\hline Any carotid atheromatous disease & 7 & $5 \%$ & 37 & $26 \%$ & $0.15(0.06-0.34)$ & $<.001^{\mathrm{a}}$ \\
\hline
\end{tabular}

Note:-FMD indicates fibromuscular dysplasia.

a Significant.

Table 4: Multivariate model for any ICA tortuosity by demographic and baseline characteristics

\begin{tabular}{lccc}
\hline & \multicolumn{3}{c}{ Any ICA Tortuosity } \\
\cline { 2 - 4 } \multicolumn{1}{c}{ Variable } & Adjusted OR & $95 \% \mathrm{CI}$ & Adjusted $\boldsymbol{P}$ \\
\hline CTD status & 5.24 & $2.78-9.90$ & $<.001^{\mathrm{a}}$ \\
HTN & 2.75 & $1.30-5.84$ & $.008^{\mathrm{a}}$ \\
Stroke history & 1.76 & $0.78-4.01$ & .18 \\
DM & 1.67 & $0.49-5.78$ & .41 \\
Dyslipidemia & 1.67 & $0.77-3.64$ & .19 \\
CAD history & 1.45 & $0.48-4.40$ & .51 \\
Age & 1.00 & $0.98-1.02$ & .99 \\
Smoking & 0.89 & $0.45-1.78$ & .65 \\
Sex (female) & 0.52 & $0.28-0.98$ & $.04^{\mathrm{a}}$ \\
\hline
\end{tabular}

Note:-CAD indicates coronary artery disease; HTN, hypertension; DM, diabetes mellitus.

${ }^{\mathrm{a}}$ Significant.

analysis of the association of demographic and clinical characteristics with any ICA tortuosity (Table 4). CTD status was highly associated with any ICA tortuosity (adjusted OR, 5.24; 95\% CI, 2.78-9.90; $P<.001)$. Additionally, hypertension (adjusted OR, 2.75; 95\% CI, 1.30-5.84; $P=.008$ ) and female sex (adjusted OR, 0.52; 95\% CI, 0.28-0.98; $P=.04$ ) were found to be significantly associated. The remaining variables, did not reach significance.

\section{Analysis by CTD Type}

Given the above findings, we sought to delineate the prevalence of carotid abnormalities within specific CTDs (On-line Table 3). Compared with the control group, patients with Marfan syndrome exhibit increased prevalence of any ICA tortuosity, loops, and coils. Patients with Marfan syndrome demonstrated marked prevalence of bilateral tortuosity (64\% versus $10 \%$; OR, 14.93; 95\% CI, 6.14-36.30; $P<.001)$. Notably, the prevalence of carotid kinking was not found to be significantly increased relative to the control population. Furthermore, analysis revealed an increased prevalence of retrojugular carotid course (48\% versus 20\%; OR, 3.70; 95\% CI, 1.67-8.19; $P<.001)$.

Patients with NF1 had an increased prevalence of any ICA tortuosity, specifically, carotid coils (15\% versus $3 \%$; OR, 6.32;
95\% CI, 1.47-27.13; $P=.005)$ relative to controls. Similarly, patients with LDS had significantly increased total vessel tortuosity with coils making up most observed abnormalities (50\% versus 3\%; OR, 34.75; 95\% CI, 8.60-140.33; $P<.001$ ). Additionally, patients with LDS had a significantly increased prevalence of bilateral carotid tortuosity compared with controls (44\% versus 10\%; OR, 6.64; 95\% CI, 2.16-20.41; $P<.001$ ). The prevalence of any vascular anomaly in patients with EDS, vascular and non-vascular types, was not significantly different from that in controls (On-line Table 3).

\section{Sensitivity Analysis}

A total of 43 patients had both aortic aneurysms and carotid tortuosity. Among patients with CTDs, 41 patients (28.7\%) had aortic aneurysms and carotid tortuosity, and among patients without CTD, 2 patients (1.4\%) had aortic aneurysms and tortuosity (OR, 28.3; 95\% CI, 6.7-119.8; $P<.001)$. The positive predictive value of the combination of aortic aneurysm or dilation and carotid tortuosity being associated with connective tissue disease was 95.4\%. The specificity was $98.6 \%$.

\section{DISCUSSION}

This study finds that the prevalence of carotid artery tortuosity is increased in CTD, particularly in patients with Marfan syndrome, LDS, and NF1. Specifically, carotid artery tortuosity was found in $44 \%$ of patients with CTD, 3-fold the prevalence in controls. Such tortuosity was frequently bilateral; $30 \%$ of all patients with CTD exhibited bilateral ICA tortuosity. Among CTDs, patients with Marfan syndrome had the highest prevalence of tortuosity, followed by LDS and NF1. Meanwhile, rates of carotid tortuosity were similar to those in controls among patients with both vascular and nonvascular types of EDS. One interesting finding from our study was that the combination of an ascending aortic aneurysm and any carotid artery tortuosity was highly associated with connective tissue disease with an odds ratio of 28 and a specificity of nearly $99 \%$. These findings are important because they indicate that carotid artery tortuosity, especially when associated with an ascending aortic aneurysm or dilation, is highly associated with connective tissue disease. 
There have been no studies, to our knowledge, evaluating the prevalence of carotid artery tortuosity among patients with CTDs outside of limited descriptive cases. ${ }^{11}$ However, carotid tortuosity has been associated with connective tissue-related pathologies, such as cervical carotid dissection. ${ }^{1,2,12}$ Additionally, retrospective studies found the prevalence of ICA tortuosity to be $53 \%-63 \%$ in patients with dissection and 20\%-34\% in controls. ${ }^{2,13}$ Most interesting, Kim et $\mathrm{al}^{2}$ further found that a retrojugular carotid course was significantly associated with dissection; 35\% of dissections were associated with a retrojugular course relative to $16 \%$ in controls. Compared with the present study demonstrating a significantly increased prevalence of retrojugular course in patients with Marfan syndrome, such a finding may help explain the risk of dissection in this patient population.

We note a stark difference in the prevalence of carotid tortuosity across CTD types. The prevalence of any ICA tortuosity was $88 \%$ in Marfan syndrome, 63\% in Loeys-Dietz syndrome, $42 \%$ in NF1, and $19 \%$ in patients with both vascular and nonvascular types of EDS. Marfan syndrome is due to a defect in the structure of fibrillin-1, while LDS is due to mutations in the transforming growth factor $\beta$ protein receptor. Loss of microfibrils as a result of a defective fibrillin-1 results in aberrant transforming growth factor $\beta$ signaling, which is essential to the proper formation of the extracellular matrix, including the biogenesis and maintenance of elastic fibers. Meanwhile, both vascular and nonvascular forms of EDS result in abnormal collagen structure rather than elastin. A previous hypothesis states that the extracranial ICA may be susceptible as a transition zone from an elastic vessel to muscular vessel. ${ }^{6,14}$ Given the higher prevalence of carotid tortuosity in Marfan syndrome and patients with LDS and the fact that the respective mutated proteins in these 2 disorders are part of the same pathway affecting the biogenesis and maintenance of elastic fibers, we hypothesize that aberrations in elastic fiber structure rather than collagen structure are greater contributors to the development of tortuous vasculature.

Notably, little is known about subtypes of carotid tortuosity and their potential downstream effects. A study using in vitro and in vivo physiologic techniques found a significant pressure differential across carotid kinks; however, whether this is significant in other forms of tortuosity is unknown. ${ }^{7,15}$ Here, carotid coils were significantly more prevalent in those with CTD $(23 \%$ versus 3\%). This finding is in line with the suggestion that carotid coils represent consequences of embryologic origins, whereas other tortuosity may be indicative of acquired risk factors. ${ }^{6}$ Notably, the definition of tortuosity itself is wide-ranging, with some studies, including ours, following the descriptive WeibelFields and Metz modified criteria and others expanding this to include linear measurements of tortuosity. ${ }^{1,2,9,16}$ Given possible differences in etiology and pathology, continued use of descriptive criteria alongside other measures is important toward evolving our understanding of tortuosity.

Numerous factors including hypertension, elevated BMI, advanced age, and atherosclerotic disease have been variably associated with tortuosity. ${ }^{3,7-9,16,17}$ Atherosclerosis and age were noted as significant risks in early studies on carotid tortuosity. ${ }^{3}$ In contrast, using sonographic assessment of 345 patients, TogayIsikay et $\mathrm{al}^{16}$ found no clear association with carotid tortuosity and stroke risk factors, including stenosis and atherosclerosis. Furthermore, a previous report suggests that hypertension is more prevalent in patients with tortuosity. ${ }^{7}$ However, a recent study ultimately failed to find such a result, instead finding body mass index as a significant factor., ${ }^{7,9}$ Other previously found associations include laterality ${ }^{1,9,16,17}$ and sex differences, ${ }^{9,12,16-18}$ though, notably, these remain discrepant and of uncertain significance. Using multivariate analysis, here we found hypertension, stroke history, and smoking history to be associated with tortuosity. We did not find an association between age, sex, diabetes, coronary artery disease and any ICA tortuosity. Contrary to previous suggestions, though tortuosity was increased in CTD, these patients had decreased carotid atherosclerosis compared with controls. Regarding laterality, we found bilateral tortuosity to be significantly increased in CTD. Additionally, right-sided predominance was noted among all CTD types, with Marfan syndrome exhibiting the most marked prevalence. Such a finding could suggest manifestation of tortuosity associated with proximity to the brachiocephalic trunk, in addition to known aortic pathologies. Outside of the aforementioned, tortuosity risk factors have been documented. Intriguing studies report carotid tortuosity in those with sickle cell anemia, thus suggesting an alternative mechanism to direct vascular structure abnormalities. Given the broad array of associations found and not found, future meta-analysis would be of benefit.

CTDs are associated with other vascular tortuosities. For example, the association of Marfan syndrome with aortic root dilation is well-established. Furthermore, recent evidence finds other cervical vasculature tortuosities in CTD. Vertebral artery tortuosity was found to be associated with earlier vascular interventions and worse outcomes in a young cohort of patients with CTD, particularly those with Marfan syndrome or LDS. ${ }^{19}$ Here, we found a significantly increased prevalence of aortic, carotid, subclavian/brachial, abdominal, and renal pathology in patients with CTD. While this finding indicates a larger proportion of vascular abnormalities in those with CTD, the extent of such an effect may be exaggerated. Follow-up and the presence of other diagnostic imaging was not controlled for in this secondary analysis. Furthermore, some forms of tortuosity may be transient. A retrospective study has found retropharyngeal carotid course to change with time across patients, thus lending evidence that pathway alterations and tortuosity are dynamic processes. ${ }^{20}$ Future prospective research exploring the temporal relationship among these risk factors, tortuosity, and ultimate disease pathology is needed.

Our study has some practical implications and also potentially paves the way for further study of connective tissue abnormalities for patients with ecstatic or dissected carotid arteries. Given the relatively high prevalence of carotid artery tortuosity in the general population and the rarity of CTDs, we certainly do not call for all patients with carotid tortuosity to be screened for CTDs. However, in the correct clinical context and in the presence of additional abnormal imaging findings (ie, dilated aortic root, dural ectasia, multiple aneurysms, multiple arterial ectasias, and so forth), carotid tortuosity could be an additional finding encouraging clinicians to think 
about the possibility of CTD. This is especially true given that we found that the presence of aortic root dilation or an aneurysm in combination with carotid artery tortuosity was highly associated with CTDs, with a specificity of nearly $99 \%$ and a positive predictive value of $>95 \%$. Regarding future studies, it may be interesting to determine the prevalence of carotid dissections in patients with CTD with and without tortuosity. It is possible that vascular tortuosity is an important phenotypic component of patients with CTD who are at risk for dissection, thus making it an indication for regular cervical vascular screening.

\section{Limitations}

This study has limitations. These include limited patients with specific CTD subtypes identified for inclusion in this study. Strict sex and age matching were not applied. This study looked at imaging across a 20 -year period with varied scanners and imaging protocols. The selection of patients with CTD with available cervical vasculature imaging may lead to incorporation bias of those with suspected disease and may not represent the larger CTD population. The case-control study design precludes the accurate determination of the incidence of CTD in those with tortuosity. Similarly, secondary data points, including the prevalence of other vascular abnormalities, may be subject to inclusion bias increased availability of other in patients with CTD relative to controls. Furthermore, inter- and intraobserver concordance of vessel tortuosity identification was not studied.

\section{CONCLUSIONS}

Tortuosity of the cervical internal carotid arteries is highly prevalent in patients with CTDs, particularly patients with Marfan syndrome or LDS. The finding of an ascending aortic aneurysm or dilation in combination with any carotid tortuosity is highly specific for the presence of a connective tissue disease. Such findings are relevant for risk assessment for vascular complications in CTD, endovascular treatment planning, and in understanding the pathomechanisms of vascular tortuosity in general.

\section{REFERENCES}

1. Saba L, Argiolas GM, Sumer S, et al. Association between internal carotid artery dissection and arterial tortuosity. Neuroradiology 2015;57:149-53 CrossRef Medline

2. Kim ST, Brinjikji W, Lehman VT, et al. Association between carotid artery tortuosity and carotid dissection: a case-control study. $J$ Neurosurg Sci 2018;62:413-17 CrossRef Medline

3. Del Corso L, Moruzzo D, Conte B, et al. Tortuosity, kinking, and coiling of the carotid artery: expression of atherosclerosis or aging? Angiology 1998;49:361-71 CrossRef Medline
4. Lam RC, Lin SC, DeRubertis B, et al. The impact of increasing age on anatomic factors affecting carotid angioplasty and stenting. $J$ Vasc Surg 2007;45:875-80 CrossRef Medline

5. Han HC. Twisted blood vessels: symptoms, etiology and biomechanical mechanisms. J Vasc Res 2012;49:185-97 CrossRef Medline

6. La Barbera G, La Marca G, Martino A, et al. Kinking, coiling, and tortuosity of extracranial internal carotid artery: is it the effect of a metaplasia? Surg Radiology Anat 2006;28:573-80 CrossRef Medline

7. Pancera P, Ribul M, Presciuttini B, et al. Prevalence of carotid artery kinking in 590 consecutive subjects evaluated by echocolor Doppler: is there a correlation with arterial hypertension? J Intern Med 2000;248:7-12 CrossRef Medline

8. Thomas JB, Antiga L, Che SL, et al. Variation in the carotid bifurcation geometry of young versus older adults: implications for geometric risk of atherosclerosis. Stroke 2005;36:2450-56 CrossRef Medline

9. Wang HF, Wang DM, Wang JJ, et al. Extracranial internal carotid artery tortuosity and body mass index. Front Neurol 2017;8:508 CrossRef Medline

10. Kim ST, Brinjikji W, Lanzino G, et al. Neurovascular manifestations of connective-tissue diseases: a review. Interv Neuroradiol 2016;22:624-37 CrossRef Medline

11. Kondo M, Itoh S, Nagano K, et al. A 10-year-old boy with Marfan syndrome exhibiting cerebrovascular abnormalities. Brain Dev 2001;23:251-54 Medline

12. Kim BJ, Yang E, Kim NY, et al. Vascular tortuosity may be associated with cervical artery dissection. Stroke 2016;47:2548-52 CrossRef Medline

13. Barbour PJ, Castaldo JE, Rae-Grant AD, et al. Internal carotid artery redundancy is significantly associated with dissection. Stroke 1994;25:1201-06 CrossRef Medline

14. Albanese V, Spadaro A, Iannotti F, et al. Elastic tissue dysplasia of coiled internal carotid artery in an adult: case report. J Neurosurg 1983;58:781-85 CrossRef Medline

15. Wang L, Zhao F, Wang D, et al. Pressure drop in tortuosity/kinking of the internal carotid artery: simulation and clinical investigation. BioMed Res Int 2016;2016:2428970 CrossRef Medline

16. Togay-Işikay C, Kim J, Betterman K, et al. Carotid artery tortuosity, kinking, coiling: stroke risk factor, marker, or curiosity? Acta Neurol Belg 2005;105:68-72 Medline

17. Macchi C, Gulisano M, Giannelli F, et al. Kinking of the human internal carotid artery: a statistical study in 100 healthy subjects by echocolor Doppler. J Cardiovasc Surg (Torino) 1997;38:629-37 Medline

18. Choudhry FA, Grantham JT, Rai AT, et al. Vascular geometry of the extracranial carotid arteries: an analysis of length, diameter, and tortuosity. J Neurointerv Surg 2016;8:536-40 CrossRef Medline

19. Morris SA, Orbach DB, Geva T, et al. Increased vertebral artery tortuosity index is associated with adverse outcomes in children and young adults with connective tissue disorders. Circulation 2011;124:388-96 CrossRef Medline

20. Lukins DE, Pilati S, Escott EJ. The moving carotid artery: a retrospective review of the retropharyngeal carotid artery and the incidence of positional changes on serial studies. AJNR Am J Neuroradiol 2016;37:336-41 CrossRef Medline 